\section{Removable subcuticular skin suture in acute appendicitis: a prospective comparative clinical trial}

Standard surgical teaching advises the use of interrupted sutures for skin closure in potentially infected wounds. ${ }^{1-3}$ Trials supporting or refuting this premise are few. So far as we are aware no trial has been performed using a removable subcuticular stitch for skin closure in acute appendicitis. We report the results of a prospective trial to compare the two methods of skin closure.

\section{Patients, methods, and results}

One hundred and eighty-four patients with acute appendicitis were entered into a prospective trial to compare skin wounds closed by a continuous subcuticular Prolene suture with those closed with interrupted Prolene sutures. Only patients with appendicitis subsequently confirmed histologically were included; perforation of the appendix did not contraindicate entry into the trial.

All skin incisions were transverse or oblique, and the abdomen was entered through standard grid-iron or transrectus approaches. Drains $(17 / 146 ; 12 \%)$ and antibiotics $(53 / 146 ; 35 \%)$ were used at the discretion of the surgeon. Dexon was used for peritoneal and muscle closure in all cases. Neither peritoneal nor wound lavage was performed and, no topical agents were applied to the wound. After closure of the muscle layer a randomly selected sealed envelope was opened to determine the choice of skin suture. The severity of the appendicitis was classified as: (a) acute inflammation, $(b)$ pus present, or $(c)$ perforation. All wounds were examined daily and again two weeks after the patient left hospital; those with a discharge were considered to be infected. Skin sutures were removed between five and seven days after operation.

Since 33 patients failed to attend for follow-up and five were excluded because of protocol violations, 146 cases were evaluated. Age ranges were comparable in the two groups of patients: 5-66 years (mean 20.9 years) for those with interrupted sutures and 4-52 years (mean 19.9 years) for those with subcuticular sutures. Wound closed with interrupted sutures and those closed with a subcuticular suture became infected in $15.9 \%$ and $15.7 \%$ respectively; when the appendix was perforated the infection rates were $23.0 \%$ and $26.3 \%$ respectively (table). Two wounds closed with an interrupted suture and one with a subcuticular suture required secondary suturing of the skin. In no case did complete wound dehiscence, postoperative intraabdominal abscess, or death occur in either group.

The cosmetic appearance of wounds closed with a subcuticular suture was noticeably better than that with interrupted sutures, since cross-scarring was absent.

Wound infection rates in patients using two methods of skin closure

\begin{tabular}{|c|c|c|c|c|c|}
\hline \multicolumn{4}{|c|}{ State of appendix } & \multirow{2}{*}{$\begin{array}{c}\text { Interrupted sutures } \\
4 / 34(11.8 \%) \\
3 / 16(18.8 \%) \\
3 / 13(23.0 \%)\end{array}$} & \multirow{2}{*}{$\begin{array}{c}\text { Continuous sutures } \\
3 / 43(7 \cdot 0 \%(\%) \\
5 / 21(23 \cdot 8,0) \\
5 / 19(26.3 \%)\end{array}$} \\
\hline $\begin{array}{l}\text { Inflamed } \\
\text { Containing pus } \\
\text { Perforated }\end{array}$ & $\begin{array}{l}\ldots \\
\cdots\end{array}$ & 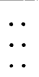 & $\begin{array}{l}\ldots \\
\cdots\end{array}$ & & \\
\hline Total & $\ldots$ & $\cdots$ & $\ldots$ & $10 / 63(15.9 \%)$ & $13 / 83(15 \cdot 7 \%)$ \\
\hline
\end{tabular}

\section{Comment}

Subcuticular sutures have become increasingly popular for skin closure of uninfected wounds. ${ }^{4}$ In appendicectomy wounds, however, subcuticular Dexon sutures are noticeably inferior to interrupted nylon sutures. ${ }^{5}$ In this study this was not the case with subcuticular Prolene sutures. The difference in infection rates between these two trials must be due to the use of Dexon rather than to the use of a subcuticular suture. The capillarity of Dexon and its prolonged presence in the skin wound may possibly predipose to infection.

One criticism of the subcuticular suture has been that when infection occurs the wound is difficult to drain and total skin dehiscence is inevitable. We have found that the stitch may be cut at any point in the wound and withdrawn the necessary $1-2 \mathrm{~cm}$ without any further dehiscence. In about $40 \%$ of wound infections the skin suture did not influence the management of infected wounds, as the suture had already been removed when the infection became apparent.

Another criticism of removable subcuticular sutures is that they can break, making complete removal difficult. This is not so if Prolene is used rather than nylon, as the friction between the suture and each skin edge is much less and continuous subcuticular sutures 6 inches in length are easily removed.

We have found that a continuous removable subcuticular Prolene suture may be used with impunity to close skin wounds after appendicectomy, and its cosmetic advantage over interrupted sutures possibly makes it the stitch of choice.

We would like to thank those consultants and junior surgeons who have given us their enthusiastic co-operation in the performance of this trial.

${ }^{1}$ Rob C, Smith R. Operative surgery, vol 5. London: Butterworth, 1969:441.

${ }^{2}$ Maingot R. Abdominal operations, vol 2. New York: Appleton-CenturyCrofts, 1974:1382.

${ }^{3}$ Farquharson E. Textbook of operative surgery. Edinburgh: Churchill Livingstone, 1978:624.

${ }^{4}$ Clough JV, Alexander-Williams J. Surgical and economic advantages of polyglycolic-acid suture material in skin closure. Lancet $1975 ; \mathrm{i}: 194-5$.

${ }^{5}$ Foster GE, Hardy EG, Hardcastle JD. Subcuticular suturing after appendicectomy. Lancet 1977; i:1128-9.

(Accepted 1 December 1981)

Walsgrave Hospital, Coventry CV2 2DX

G B HOPKINSON, FRCS, surgical registrar

B R BULLEN, FRCs, consultant surgeon

\section{Tamoxifen as initial sole treatment of localised breast cancer in elderly women: a pilot study}

Breast lumps that develop in elderly women are most likely to be mammary carcinomas. ${ }^{1}$ Often such women have never been to hospital and the prospect of admission is alarming to them. The presence of intercurrent illnesses in women aged 70 years and over who develop breast cancer is, as might be expected, extremely high, increasing the risk of anaesthesia and surgery. ${ }^{2}$ These patients often ask whether, instead of being excised, their breast lumps can be "dispersed." In a four-year pilot study we treated elderly women with apparently localised breast cancer with tamoxifen in view of its proved efficacy in advanced breast cancer in this age group. ${ }^{3}$

\section{Patients, methods, and results}

A consecutive series of 67 women aged 75 years or over with cytologically confirmed carcinoma of the breast that had been shown by blood tests and limited metestatic surveys to be localised were prescribed tamoxifen in doses of 10 to $20 \mathrm{mg}$ twice daily. Also included were a few younger patients with intercurrent disease. Before treatment each patient was weighed and the tumour measured in two dimensions with callipers. These measurements and a check on platelets and calcium were repeated at follow-up visits, which were monthly for the first three months and thereafter at intervals of three months, then six months.

The 67 patients studied had a mean age of 78.3 years (range 67-95). Two further patients declined to participate, preferring surgery. No patient was excluded because of side effects or adverse changes in the results of the blood tests. Forty-nine patients $(73 \%)$ responded sufficiently to persist with treatment beyond the first follow-up visits (table). The response rate was similar for both stages II and stage III tumours, but the time to become impalpable was longer for the larger lesions. Almost all the tumours that eventually responded showed signs of response after one month of treatment. More than 12 months were required by nine $(18 \%)$ to achieve their ultimate response. Tamoxifen was continued for a few static lesions since there was no evidence of either local or systemic progression. All the patients tolerated the drug well.

In 18 patients tamoxifen failed from the outset. In nine of these tumour size was static: in five at one month, in three at six months, and in one at 36 months. Increase in measured size of the primary tumour occurred in nine women, in three of whom this was gradual. Three further failures occurred : one tumour recurred after complete remission for 12 months, and in two cases partial remission was followed by local progression of disease. Twelve of the "failed group" went on to mastectomy. One of these died of pulmonary embolism 10 days after operation. Nodal histology was obtained in 10 patients, and was negative in eight.

\section{Comment}

Our chief consideration in using tamoxifen as initial sole treatment for localised carcinoma of the breast in older women was to attempt to spare them surgery and admission to hospital. The presence of a high incidence of intercurrent, usually chronic problems and the rela- 